\title{
On the Question of + $P$-Invariance
}

\section{and a Possible Difference of $\theta_{v}$ and $\theta_{A}$ in Hyperon Leptonic Decays.}

\section{W. DRECHRLER}

Institut für Hochenergiephysik, Tniversität Heidelberg - Heidelberg

(Nuoro Cimento, 38, $345(1965))$

Read $k_{0}^{\top}=\left(m_{\mathrm{A}}^{2}-m_{\mathrm{B}}^{2}\right) / 2 m_{\mathrm{A}}$ on page 350 (second line from the bottom).

Formula (17) should read:

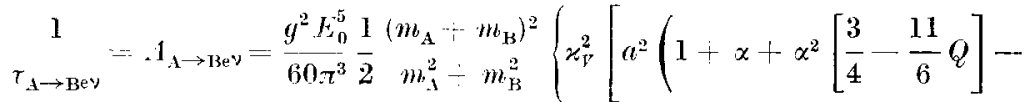

$$
\begin{aligned}
& \left.-x^{3}\left[\frac{1}{4}-\frac{1}{3} Q\right]-(1+\alpha) \frac{4}{9} Q<r^{2} ; E_{0}^{2}\right)+ \\
& \left.-a b\left(\alpha^{2}\left[1-\frac{3}{3} Q\right]+\alpha^{3}\left[\frac{1}{2}-\frac{1}{3} Q\left(\frac{9}{2}+\frac{m_{\mathrm{B}}}{m_{\mathrm{A}}}\right)\right]\right)+b^{2}\left(\alpha^{2}+\alpha^{3}\right)\left[\frac{1}{2}--\frac{5}{3} Q\right]\right]+ \\
& +x_{-A}^{2}\left[c^{2}\left(3+\alpha+\left(\alpha^{3}-x^{3}\right)\left[\frac{1}{4}-\frac{1}{3} Q\right]--(11+2 x) \frac{2}{9} Q<r^{\prime 2}>E_{0}^{\prime 2}\right)-\right. \\
& -c \operatorname{Re} d\left(2 \alpha+\alpha^{2}\left[1-\frac{8}{3} Q-\frac{11}{3} \frac{m_{\mathrm{B}}}{m_{\mathrm{A}}} Q\right]-\alpha^{3} \frac{m_{\mathrm{B}}}{3 m_{\mathrm{A}}} Q-2 \alpha Q<r^{2} ; E_{0}^{2}\right)+ \\
& \left.\left.+|d|^{2}\left(\alpha^{2}\left[\frac{1}{2}-\frac{1}{3} Q\right]+\alpha^{3}\left[\frac{1}{2}-\frac{5}{3} Q\right]\right)\right]\right\} \text {. }
\end{aligned}
$$

With $\theta_{V}=0.214 \pm 0.010$ and the now accepted value $g_{A} / g_{V}=-1.18 \pm 0.02$ one obtains the solutions:

Sol. I $\quad \theta_{A}=0.24_{-0.11}^{+0.26}, \quad \xi=0.61_{-0.03}^{+0.11}, \quad$ Re $\mu=+8_{-19}^{+12}$,

Sol. I' $\theta_{A}=0.35 \pm 0.03, \quad \xi=0.69 \pm 0.03, \quad \operatorname{Re} \mu=\operatorname{Im} \mu=0$,

Sol. II $\quad \theta_{A}=0.24_{-0.13}^{+0.12}, \quad \xi=0.65_{-0.09}^{+0.07}, \quad \vdots \operatorname{Im} \mu \mid=19_{-19}^{+14}$. 